\title{
Reseña de Handbook of Research on Citizenship and Heritage Education
}

Review of Handbook of Research on Citizenship and Heritage Education

Mónica Trabajo Rite*

Editores: Emilio José Delgado-Algarra y José María Cuenca López

Título: Handbook of Research on Citizenship and Heritage Education

Edición: IGI Global

Lugar de publicación: Hershey (PA, Estados Unidos)

Año: 2020

Idioma: Inglés

ISSN: 2326-8905 / EISSN: 2326-8913

Páginas: 622

El libro, editado por Emilio José Delgado y José María Cuenca se presenta como un manual de referencia que une los campos de conocimiento de educación para la ciudadanía y educación patrimonial en contexto nacional e internacional. Con 23 capítulos, distribuidos en 3 secciones, recoge temáticas relacionadas con la ciudadanía democrática, el patrimonio cultural, las políticas educativas o el diseño curricular, incluyendo contenidos relacionados con la docencia escolar en ciencias sociales y la formación docente. Las investigaciones que se describen están enfocadas hacia la promoción de la participación ciudadana y la transformación de la sociedad.

Emilio José Delgado y José $\mathrm{M}^{\mathrm{a}}$ Cuenca abren la primera sección con el capítulo "Challenges for the Construction of Identities With Historical Consciousness: Heritage Education and Citizenship Education". Relacionan los concpetos de educación patrimonial y educación para la ciudadanía en una revisión bibliográfica. Presentan experiencias de innovación y definen líneas de investigación relacionadas con temáticas socioculturalmente relevantes. "Critical Citizenship Education and Heritage Education”, es el capítulo 2 presentado por Antoni Santisteban, Neus González y Joan Pagès. Parten de la educación patrimonial como disciplina mediante la cual desarrollar

\footnotetext{
*Universidad de Huelva • ORCID 0000-0003-0641-8371• monica.trabajo@ddcc.uhu.es

Trabajo, M. (2020). Reseña de Handbook of Research on Citizenship and Heritage Education. CLIO. History and History teaching, 46, 327-331. https://doi.org/10.26754/ojs clio/clio.2020465296. Recibido: 27/9/2020. Aceptado: 3/11/2020.
} 
conciencia histórica. Abordan el patrimonio como evidencia de cambio y continuida que beneficia la comprensión de la evolución por parte de la ciudadanía con conciencia crítica. El capítulo 3 titulado "Heritage in Conflict: A Way to Educate in a Critical and Participative Citizenship", Myriam Martín y Jesús Estepa realizan un recorrido por las controversias que surgen en torno al patrimonio en la búsqueda de estrategias para abordarlo a partir de cuestiones socioambientales relevantes.

Catherine Broom presenta el capítulo "Citizenship and Social Studies Curricula in British Columbia, Canada: Contemporary Realities and Alternative Possibilities". La autora revisa los planes de estudio de estudios sociales en Columbia Británica en relación con la educación ciudadana y concluye planteando la necesidad de elaboración de planes de estudio alternativos en los que se promueva la enseñanza de las ideas en la formación de la ciudadanía y los estudios sociales. En el capítulo 5 "Heritage Education and Global Citizenship", Gustavo González-Valencia, Mariona Massip y Jordi Castellví, realizan una revisión documental sobre la identidad local, nacional y global en educación formal y no formal y su relación con la formación ciudadana. Ainoa Escribano, Pedro Miralles y Francisca Serrano, en el capítulo "Heritage and Museums as Objects of Education for Citizenship in the Teaching of History", contemplan el patrimonio arqueológico como un recurso en la formación histórica y ciudadana. Laia Coma, Concha Fuentes y Lydia Sánchez presentan el capítulo "Heritage Education as an Instrument for the Construction of a Democratic Citizenship in the Social Sciences Classrooms of Secondary Education". Reflexionan sobre la importancia de la educación patrimonial como símbolo de identidad en las aulas de secundaria en España en favor de la construcción de una sociedad democrática. En el capítulo 8 "Arabic Language Heritage Schools: The Educational Potential of Celebrating Identity and Diversity", Beatrice Szczepek, Geraldine Bengsch, Fatma Said, Jayme Scally e lan Davies, trabajan juntos en una investigación empírica realizada en escuelas de lengua árabe de Reino Unido. Pretenden poner en valor la identidad personal y social y otorgar una respuesta educativa a la diferencia cultural y poner fin a la amenaza percibida por el reclutamiento de grupos terroristas. Olaia Fontal y Marta Martínez cierran la primera sección con el capítulo "The Heritage and Education Research Network: Place Value on Cultural Heritage in Europe". Las autoras proponen la creación de una red interdisciplinar que integre a múltiples agentes involucrados en la gestión del patrimonio para investigar el enfoque otorgado 
a la educación patrimonial en diferentes países europeos, analizando conceptos, prácticas y experiencias.

La segunda sección presenta trabajos de investigación en ámbito internacional. El capítulo 10 "Heritage Education as a Tool for Creating Critical Citizens: Analysis of Conceptions of Teachers in Training", presentado por Mario Ferreras, José Antonio Pineda y Coral Hunt, describen varios programas y proyectos de la Comisión Europea con el objetivo de difundir los valores democráticos y sensibilizar sobre el poder de la educación y su papel en la creación de una ciudadanía democrática y participativa. A continuación, Wing On Lee, Nan Hao y Qian Zhou en el capítulo "Heritage Education in Central China: Agendas for Cultural and Inter-Cultural Citizenship Education" revisan el nexo de unión entre educación patrimonial y formación ciudadana incluyendo la educación cultural e intercultural. Bajo un estudio de caso en Las Llanuras Centrales de China, matizan que el pasado de la China Central no solo es "Cultural" sino también "Intercultural". En el capítulo 12 "Trainee Teachers' Perceptions of History Teaching and the Critical Education of Citizenship", Cosme Gómez-Carrasco, Ramón López-Facal y Belén Castro analizan las percepciones de los docentes en formación de educación primaria sobre la enseñanza de la historia. Los resultados muestran las disonancias entre la identificación de los docentes relacionada con un modelo crítico que utiliza métodos de enseñanza activos y promueve valores éticos relacionados con la justicia social, con un modelo implícito tradicional relacionado con sus concepciones teóricas y metodológicas. Los autores concluyen en la necesidad de vincular la formación hacia la práctica. Un grupo de investigación de Chile da forma al capítulo "Heritage Education and Citizen Education in Chile". Se presentan los resultados del análisis de la praxis de diez museos chilenos cuyo objetivo es describir y analizar el vínculo existente entre el museo y la escuela. Iratxe Gillate, Úrsula Luna, Janire Castrillo y Álex Ibáñez en el capítulo "Historical Memory in Heritage Education Apps: A Resource for Building Social and Civic Competence.", analizan aplicaciones destinadas a preservar la memoria histórica y que se presentan como recurso para promover la competencia social y ciudadana. Beatrice Borghi y Filippo Galletti, presentan el trabajo "Researches, Projects, and Experiences in Didactics of History and Heritage From the DiPaSt Center of the University of Bologna, Italy". Los autores presentan las investigaciones más representativas lideradas por el Centro Internacional de Didáctica de la Historia y el Patrimonio de la Universidad de Bolonia 
(Italia) en el que participan escuelas y profesores diversos niveles, instituciones y autoridades locales. En el capítulo 16 "Religious and Ethnic Identification of Minoritized Youth in Hong Kong: Exploring Acculturation Outcomes" Hin Wah Chris Cheung, Miron K Bhowmik, Kerry J Kennedy, Hoi Yu Ng y Ming Tak Hue exploran diversos casos de migrantes paquistaníes e indios en Hong Kong con el objetivo de analizar el proceso de aculturación. Concluyen en que no se puede generalizar ya que, la aculturación es un proceso con muchos matices y depende del contexto. Sebastián Molina y María del Pilar Riquelme protagonizan el último capítulo de la sección 2, "Future Citizens: Analysis of the Knowledge and Attitudes on Citizenship of Elementary Education Pupils of Murcia, Spain From an Inquiry Based on the ICCS". Los autores analizan los conocimientos, comportamientos, actitudes y valores de los estudiantes españoles en el último año de educación primaria de un colegio público en relación con la ciudadanía crítica y participativa y el civismo. La tercera sección incluye experiencias y propuestas didácticas innovadoras desde una perspectiva internacional. Antonio Brusa en el capítulo "The Difficulty of Teaching Historical Landscape: Observations Starting From the Italian Situation", relaciona identidad y antimodernidad como conceptos fundamentales para comprender el paisaje histórico en las escuelas italianas. Defienden la deconstrucción de estereotipos para configurar la didáctica de la historia. Julia Gantenberg y Marc Partetzke presentan "Democratic Learning Environment Formats for the Empowerment of Youth". Señalan las implicaciones a nivel europeo de la radicalización y las tendencias antidemocráticas locales. Defienden la creación de programas educativos que incrementen el compromiso y la participación de jóvenes en el contexto de una sociedad civil. El capítulo "The Spanish Civil War in the Classrooms: Working for Citizenship Education" presentado por María Feliú y Xavier Hernàndez, da a conocer el potencial pedagógico de la historia de la Guerra Civil española y su patrimonio para desarrollar un programa de educación ciudadana basado en el uso de materiales patrimoniales. Pilar Rivero, Iñaki Navarro y Borja Aso, en su trabajo "Educommunication Web 2.0 for Heritage: A View From Spanish Museums", examinan cómo las grandes instituciones generan un contenido cotidiano multiforme y atractivo pero que no fomenta el intercambio de experiencias y opiniones entre los usuarios. "Conflictive Memory and Heritage Education in the Initial Training of Primary Teachers" es el capítulo de Andrés Domínguez-Almansa, Tania Riveiro, José Monteagudo y Ramón López-Facal. El estudio muestra cómo trabajando con pasados incómodos y tomándolo como un problema cívico y educativo, se inició un proceso que 
integra emoción y racionalidad crítica para dar sentido a la experiencia educativa de los estudiantes. Cierra el libro el capítulo "Educating Multicultural Citizens From a Confucian Heritage: Examples From Singapore". Sandra Pinhui, Charlene Tan y Connie S.L. Ng exploran la educación de ciudadanos multiculturales en Singapur basándose en las enseñanzas del confucianismo. 